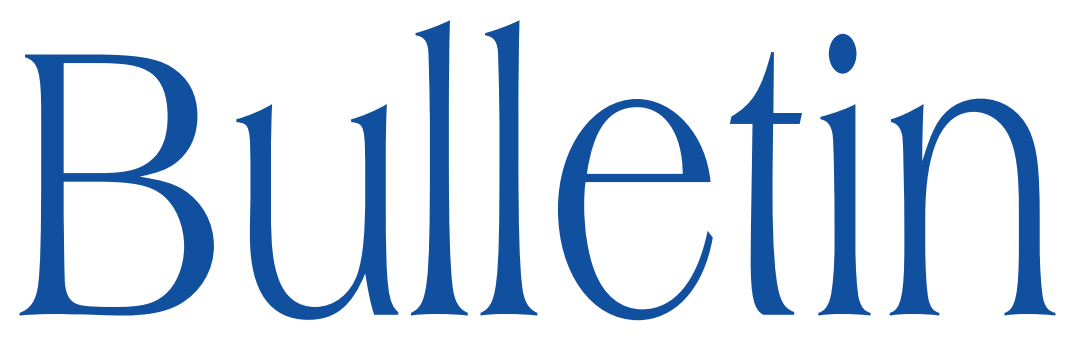

de la SOCIÉTÉ MATHÉMATIQUE DE FRANCE

\title{
SEMISTABILITY OF FROBENIUS DIRECT IMAGES
}

\section{Vikram B. Mehta \& Christian Pauly}

\section{Tome 135} Fascicule 1 


\title{
SEMISTABILITY OF FROBENIUS DIRECT IMAGES OVER CURVES
}

\author{
By Vikram B. Mehta \& Christian Pauly
}

\begin{abstract}
Let $X$ be a smooth projective curve of genus $g \geq 2$ defined over an algebraically closed field $k$ of characteristic $p>0$. Given a semistable vector bundle $E$ over $X$, we show that its direct image $F_{*} E$ under the Frobenius map $F$ of $X$ is again semistable. We deduce a numerical characterization of the stable rank- $p$ vector bundles $F_{*} L$, where $L$ is a line bundle over $X$.

Résumé (Semi-stabilité des images directes par Frobenius sur les courbes)

Soit $X$ une courbe projective lisse de genre $\geq 2$ définie sur un corps $k$ algébriquement clos de caractéristique $p>0$. Étant donné un fibré vectoriel semi-stable $E$ sur $X$, nous montrons que l'image directe $F_{*} E$ par le morphisme de Frobenius $F$ de $X$ est aussi semi-stable. Nous déduisons une caractérisation numérique du fibré vectoriel stable $F_{*} L$ de rang $p$, où $L$ est un fibré en droites sur $X$.
\end{abstract}

\section{Introduction}

Let $X$ be a smooth projective curve of genus $g \geq 2$ defined over an algebraically closed field $k$ of characteristic $p>0$ and let $F: X \rightarrow X_{1}$ be the relative $k$-linear Frobenius map. It is by now a well-established fact that on

Texte reçu le 24 janvier 2006, révisé le 21 juillet 2006

Vikram B. Menta, Tata Institute of Fundamental Research, Homi Bhabha Road, Mumbai 400005 (India) - E-mail : vikram@math.tifr.res.in

Christian Pauly, Université de Montpellier II, Département de Mathématiques, Case Courrier 051, Place Eugène Bataillon, 34095 Montpellier Cedex 5 (France)

E-mail : pauly@math.univ-montp2.fr

2000 Mathematics Subject Classification. - 14H40, 14D20, 14H40.

Key words and phrases. - Vector bundle, semistability, Frobenius. 
any curve $X$ there exist semistable vector bundles $E$ such that their pull-back under the Frobenius map $F^{*} E$ is not semistable [4, 5]. In order to control the degree of instability of the bundle $F^{*} E$, one is naturally lead (through adjunction $\left.\operatorname{Hom}_{\mathcal{O}_{X}}\left(F^{*} E, E^{\prime}\right)=\operatorname{Hom}_{\mathcal{O}_{X_{1}}}\left(E, F_{*} E^{\prime}\right)\right)$ to ask whether semistability is preserved by direct image under the Frobenius map. The answer is (somewhat surprisingly) yes. In this note we show the following result.

THEOREM 1.1. - Assume that $g \geq 2$. If $E$ is a semistable vector bundle over $X$ (of any degree), then $F_{*} E$ is also semistable.

Unfortunately we do not know whether also stability is preserved by direct image under Frobenius. It has been shown that $F_{*} L$ is stable for a line bundle $L$ (see [4, Proposition 1.2]) and that in small characteristics the bundle $F_{*} E$ is stable for any stable bundle $E$ of small rank (see [3]). The main ingredient of the proof is Faltings' cohomological criterion of semistability. We also need the fact that the generalized Verschiebung $V$, defined as the rational map from the moduli space $\mathcal{M}_{X_{1}}(r)$ of semistable rank- $r$ vector bundles over $X_{1}$ with fixed trivial determinant to the moduli space $\mathcal{M}_{X}(r)$ induced by pull-back under the relative Frobenius map $F$,

$$
V_{r}: \mathcal{M}_{X_{1}}(r) \rightarrow \mathcal{M}_{X}(r), \quad E \longmapsto F^{*} E
$$

is dominant for large $r$. We actually show a stronger statement for large $r$.

Proposition 1.2. - If $\ell \geq g(p-1)+1$ and $\ell$ prime, then the generalized Verschiebung $V_{\ell}$ is generically étale for any curve $X$. In particular $V_{\ell}$ is separable and dominant.

As an application of Theorem 1.1 we obtain an upper bound of the rational invariant $\nu$ of a vector bundle $E$, defined as

$$
\nu(E):=\mu_{\max }\left(F^{*} E\right)-\mu_{\min }\left(F^{*} E\right),
$$

where $\mu_{\max }\left(\right.$ resp. $\left.\mu_{\min }\right)$ denotes the slope of the first (resp. last) piece in the Harder-Narasimhan filtration of $F^{*} E$.

Proposition 1.3. - For any semistable rank-r vector bundle $E$

$$
\nu(E) \leq \min ((r-1)(2 g-2),(p-1)(2 g-2)) .
$$

We note that the inequality $\nu(E) \leq(r-1)(2 g-2)$ was proved in [10, Corollary 2], and in [11, Theorem 3.1]. We suspect that the relationship between both inequalities comes from the conjectural fact that the length (= number of pieces) of the Harder-Narasimhan filtration of $F^{*} E$ is at most $p$ for semistable $E$.

Finally we show that direct images of line bundles under Frobenius are characterized by maximality of the invariant $\nu$. 
Proposition 1.4. - Let $E$ be a stable rank-p vector bundle over $X$. Then the following statements are equivalent.

1) There exists a line bundle $L$ such that $E=F_{*} L$.

2) $\nu(E)=(p-1)(2 g-2)$.

We do not know whether the analogue of this proposition remains true for higher rank.

\section{Reduction to the case $\mu(E)=g-1$}

In this section we show that it is enough to prove Theorem 1.1 for semistable vector bundles $E$ with slope $\mu(E)=g-1$.

Let $E$ be a semistable vector bundle over $X$ of rank $r$ and let $s$ be the integer defined by the equality

$$
\mu(E)=g-1+\frac{s}{r} .
$$

Applying the Grothendieck-Riemann-Roch theorem to the Frobenius map $F: X \rightarrow X_{1}$, we obtain

$$
\mu\left(F_{*} E\right)=g-1+\frac{s}{p r} .
$$

Let $\pi: \widetilde{X} \rightarrow X$ be a connected étale covering of degree $n$ and let $\pi_{1}: \widetilde{X}_{1} \rightarrow X_{1}$ denote its twist by the Frobenius of $k$ (see [9, Section 4]). The diagram

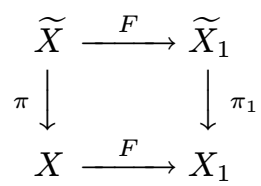

is Cartesian and we have an isomorphism

$$
\pi_{1}^{*}\left(F_{*} E\right) \cong F_{*}\left(\pi^{*} E\right) .
$$

Since semistability is preserved under pull-back by a separable morphism of curves, we see that $\pi^{*} E$ is semistable. Moreover if $F_{*}\left(\pi^{*} E\right)$ is semistable, then $F_{*} E$ is also semistable.

Let $L$ be a degree $d$ line bundle over $\widetilde{X}_{1}$. The projection formula

$$
F_{*}\left(\pi^{*} E \otimes F^{*} L\right)=F_{*}\left(\pi^{*} E\right) \otimes L
$$

shows that semistability of $F_{*}\left(\pi^{*} E\right)$ is equivalent to semistability of

$$
F_{*}\left(\pi^{*} E \otimes F^{*} L\right) .
$$


Let $\tilde{g}$ denote the genus of $\widetilde{X}$. By the Riemann-Hurwitz formula, one has $\tilde{g}-1=n(g-1)$. We compute

$$
\mu\left(\pi^{*} E \otimes F^{*} L\right)=n(g-1)+n \frac{s}{r}+p d=\tilde{g}-1+n \frac{s}{r}+p d,
$$

which gives

$$
\mu\left(F_{*}\left(\pi^{*} E \otimes F^{*} L\right)\right)=\tilde{g}-1+n \frac{s}{p r}+d .
$$

LEMma 2.1. - For any integer $m$ there exists a connected étale covering $\pi$ : $\widetilde{X} \rightarrow X$ of degree $n=p^{k} m$ for some $k$.

Proof. - If the $p$-rank of $X$ is nonzero, the statement is clear. If the $p$-rank is zero, we know by [9, Corollaire 4.3.4], that there exist connected étale coverings $Y \rightarrow X$ of degree $p^{t}$ for infinitely many integers $t$ (more precisely for all $t$ of the form $(\ell-1)(g-1)$ where $\ell$ is a large prime). Now we decompose $m=p^{s} u$ with $p$ not dividing $u$. We then take a covering $Y \rightarrow X$ of degree $p^{t}$ with $t \geq s$ and a covering $\widetilde{X} \rightarrow Y$ of degree $u$.

Now the lemma applied to the integer $m=p r$ shows existence of a connected étale covering $\pi: \widetilde{X} \rightarrow X$ of degree $n=p^{k} m$. Hence $n \frac{s}{p r}$ is an integer and we can take $d$ such that $n \frac{s}{p r}+d=0$.

To summarize, we have shown that for any semistable $E$ over $X$ there exists a covering $\pi: \widetilde{X} \rightarrow X$ and a line bundle $L$ over $\widetilde{X}_{1}$ such that the vector bundle $\widetilde{E}:=\pi^{*} E \otimes F^{*} L$ is semistable with $\mu(\widetilde{E})=\tilde{g}-1$ and such that semistability of $F_{*} \widetilde{E}$ implies semistability of $F_{*} E$.

\section{Proof of Theorem 1.1}

In order to prove semistability of $F_{*} E$ we shall use the cohomological criterion of semistability due to Faltings [2].

Proposition 3.1 (see [6, Théorème 2.4]). — Let $E$ be a rank-r vector bundle over $X$ with $\mu(E)=g-1$ and $\ell$ an integer $>\frac{1}{4} r^{2}(g-1)$. Then $E$ is semistable if and only if there exists a rank- $\ell$ vector bundle $G$ with trivial determinant such that

$$
h^{0}(X, E \otimes G)=h^{1}(X, E \otimes G)=0 .
$$

Moreover if the previous condition holds for one bundle $G$, it holds for a general bundle by upper semicontinuity of the function $G \mapsto h^{0}(X, E \otimes G)$.

REMARK. - The proof of this proposition (see [6, Section 2.4]) works over any algebraically closed field $k$. 
By Proposition 1.2 (proved in Section 4) we know that $V_{\ell}$ is dominant when $\ell$ is a large prime number. Hence a general vector bundle $G \in \mathcal{M}_{X}(\ell)$ is of the form $F^{*} G^{\prime}$ for some $G^{\prime} \in \mathcal{M}_{X_{1}}(\ell)$. Consider a semistable $E$ with $\mu(E)=g-1$. Then by Proposition $3.1 h^{0}(X, E \otimes G)=0$ for general $G \in \mathcal{M}_{X}(\ell)$. Assuming $G$ general, we can write $G=F^{*} G^{\prime}$ and we obtain by adjunction

$$
h^{0}\left(X, E \otimes F^{*} G^{\prime}\right)=h^{0}\left(X_{1}, F_{*} E \otimes G^{\prime}\right)=0 .
$$

This shows that $F_{*} E$ is semistable by Proposition 3.1.

\section{Proof of Proposition 1.2}

According to [7, Section 2], it will be enough to prove the existence of a stable vector bundle $E \in \mathcal{M}_{X_{1}}(\ell)$ satisfying $F^{*} E$ stable and

$$
h^{0}\left(X_{1}, B \otimes \operatorname{End}_{0}(E)\right)=0,
$$

because the vector space $H^{0}\left(X_{1}, B \otimes \operatorname{End}_{0}(E)\right)$ can be identified with the kernel of the differential of $V_{\ell}$ at the point $E \in \mathcal{M}_{X_{1}}(\ell)$. Here $B$ denotes the sheaf of locally exact differentials over $X_{1}$ (see [9, Section 4]).

Let $\ell \neq p$ be a prime number and let $\alpha \in J X_{1}[\ell] \cong J X[\ell]$ be a nonzero $\ell$-torsion point. We denote by

$$
\pi: \widetilde{X} \longrightarrow X \text { and } \pi_{1}: \widetilde{X}_{1} \longrightarrow X_{1}
$$

the associated cyclic étale cover of $X$ and $X_{1}$ and by $\sigma$ a generator of the Galois group $\operatorname{Gal}(\widetilde{X} / X)=\mathbb{Z} / \ell \mathbb{Z}$. We recall that the kernel of the Norm map

$$
\mathrm{Nm}: J \widetilde{X} \longrightarrow J X
$$

has $\ell$ connected components and we denote by

$$
i: P:=\operatorname{Prym}(\widetilde{X} / X) \subset J \widetilde{X}
$$

the associated Prym variety, i.e., the connected component containing the origin. Then we have an isogeny

$$
\pi^{*} \times i: J X \times P \longrightarrow J \widetilde{X}
$$

and taking direct image under $\pi$ induces a morphism

$$
P \longrightarrow \mathcal{M}_{X}(\ell), \quad L \longmapsto \pi_{*} L .
$$

Similarly we define the Prym variety $P_{1} \subset J X_{1}$ and the morphism $P_{1} \rightarrow$ $\mathcal{M}_{X_{1}}(\ell)$ (obtained by twisting with the Frobenius of $k$ ). Note that $\pi_{1 *} L$ is semistable for any $L \in P_{1}$ and stable for general $L \in P_{1}$ (see e.g., [1]). Since $F^{*}\left(\pi_{1 *} L\right) \cong \pi_{*}\left(F^{*} L\right)$ - see diagram (2.1) - and since $F^{*}$ induces the Verschiebung $V_{P}: P_{1} \rightarrow P$, which is surjective, we obtain that $\pi_{1 *} L$ and $F^{*}\left(\pi_{1 *} L\right)$ are stable for general $L \in P_{1}$. 
Therefore Proposition 1.2 will immediately follow from the next proposition. Proposition 4.1. - If $\ell \geq g(p-1)+1$ then there exists a cyclic degree $\ell$ étale cover $\pi_{1}: \widetilde{X}_{1} \rightarrow X_{1}$ with the property that

$$
h^{0}\left(X_{1}, B \otimes \operatorname{End}_{0}\left(\pi_{1 *} L\right)\right)=0
$$

for general $L \in P_{1}$.

Proof. — By relative duality for the étale map $\pi_{1}$ we have $\left(\pi_{1 *} L\right)^{*} \cong \pi_{1 *} L^{-1}$. Therefore

$$
\operatorname{End}\left(\pi_{1 *} L\right) \cong \pi_{1 *} L \otimes \pi_{1 *} L^{-1} \cong \pi_{1 *}\left(L^{-1} \otimes \pi_{1}^{*} \pi_{1 *} L\right)
$$

by the projection formula. Moreover since $\pi_{1}$ is Galois étale we have a direct sum decomposition

$$
\pi_{1}^{*} \pi_{1 *} L \cong \bigoplus_{i=0}^{\ell-1}\left(\sigma^{i}\right)^{*} L .
$$

Putting these isomorphisms together we find that

$$
\begin{aligned}
H^{0}\left(X_{1}, B \otimes \operatorname{End}\left(\pi_{1 *} L\right)\right) & =H^{0}\left(X_{1}, B \otimes \pi_{1 *}\left(\bigoplus_{i=0}^{\ell-1} L^{-1} \otimes\left(\sigma^{i}\right)^{*} L\right)\right. \\
& =\bigoplus_{i=0}^{\ell-1} H^{0}\left(X_{1}, B \otimes \pi_{1 *}\left(L^{-1} \otimes\left(\sigma^{i}\right)^{*} L\right)\right) \\
& =H^{0}\left(X_{1}, B \otimes \pi_{1 *} \mathcal{O}_{\tilde{X}_{1}}\right) \\
& \oplus \bigoplus_{i=1}^{\ell-1} H^{0}\left(X_{1}, B \otimes \pi_{1 *}\left(L^{-1} \otimes\left(\sigma^{i}\right)^{*} L\right)\right) .
\end{aligned}
$$

Moreover $\pi_{*} \mathcal{O}_{\tilde{X}_{1}}=\bigoplus_{i=0}^{\ell-1} \alpha^{i}$, which implies that

$$
\begin{aligned}
H^{0}\left(X_{1}, B \otimes \operatorname{End}_{0}\left(\pi_{1 *} L\right)\right)=\bigoplus_{i=1}^{\ell-1} H^{0}\left(X_{1}, B \otimes \alpha^{i}\right) \\
\oplus \bigoplus_{i=1}^{\ell-1} H^{0}\left(X_{1}, B \otimes \pi_{1 *}\left(L^{-1} \otimes\left(\sigma^{i}\right)^{*} L\right)\right) .
\end{aligned}
$$

Let us denote for $i=1, \ldots, \ell-1$ by $\phi_{i}$ the isogeny

$$
\phi_{i}: P_{1} \longrightarrow P_{1}, \quad L \longmapsto L^{-1} \otimes\left(\sigma^{i}\right)^{*} L .
$$

Since the function $L \mapsto h^{0}\left(X_{1}, B \otimes \operatorname{End}_{0}\left(\pi_{1 *} L\right)\right)$ is upper semicontinuous, it will be enough to show the existence of a cover $\pi_{1}: \widetilde{X}_{1} \rightarrow X_{1}$ satisfying

1) for $i=1, \ldots, \ell-1, h^{0}\left(X_{1}, B \otimes \alpha^{i}\right)=0$ (or equivalently, $P$ is an ordinary abelian variety);

2) for $M$ general in $P, h^{0}\left(X_{1}, B \otimes \pi_{1 *} M\right)=0$. 
Note that these two conditions implie that the vector space (4.1) equals $\{0\}$ for general $L \in P_{1}$, because the $\phi_{i}$ 's are surjective.

We recall that $\operatorname{ker}\left(\pi_{1}^{*}: J X_{1} \rightarrow J \widetilde{X}_{1}\right)=\langle\alpha\rangle \cong \mathbb{Z} / \ell \mathbb{Z}$ and that

$$
P_{1}[\ell]=P_{1} \cap \pi_{1}^{*}\left(J X_{1}\right) \cong \alpha^{\perp} /\langle\alpha\rangle
$$

where $\alpha^{\perp}=\left\{\beta \in J X_{1}[\ell]\right.$ with $\left.\omega(\alpha, \beta)=1\right\}$ and $\omega: J X_{1}[\ell] \times J X_{1}[\ell] \rightarrow \mu_{\ell}$ denotes the symplectic Weil form. Consider a $\beta \in \alpha^{\perp} \backslash\langle\alpha\rangle$. Then $\pi_{1}^{*} \beta \in P_{1}[\ell]$ and

$$
\pi_{1 *} \pi_{1}^{*} \beta=\bigoplus_{i=0}^{\ell=1} \beta \otimes \alpha^{i} .
$$

Again by upper semicontinuity of the function $M \mapsto h^{0}\left(X_{1}, B \otimes \pi_{1 *} M\right)$ one observes that conditions 1 ) and 2) are satisfied because of the following lemma (take $\left.M=\pi_{1}^{*} \beta\right)$.

LEMmA 4.2. - If $\ell \geq g(p-1)+1$ then there exists $(\alpha, \beta) \in J X_{1}[\ell] \times J X_{1}[\ell]$ satisfying

1) $\alpha \neq 0$ and $\beta \in \alpha^{\perp} \backslash\langle\alpha\rangle$;

2) for $i=1, \ldots, \ell-1, h^{0}\left(X_{1}, B \otimes \alpha^{i}\right)=0$;

3) for $i=0, \ldots, \ell-1, h^{0}\left(X_{1}, B \otimes \beta \otimes \alpha^{i}\right)=0$.

Proof. - We adapt the proof of [9, Lemme 4.3.5]. We denote by $\mathbb{F}_{\ell}$ the finite field $\mathbb{Z} / \ell \mathbb{Z}$. Then there exists a symplectic isomorphism $J X_{1}[\ell] \cong \mathbb{F}_{\ell}^{g} \times \mathbb{F}_{\ell}^{g}$, where the latter space is endowed with the standard symplectic form. Note that composition is written multiplicatively in $J X_{1}[\ell]$ and additively in $\mathbb{F}_{\ell}^{2 g}$. A quick computation shows that the number of isotropic 2-planes in $\mathbb{F}_{\ell}^{g} \times \mathbb{F}_{\ell}^{g}$ equals

$$
N(\ell)=\frac{\left(\ell^{2 g}-1\right)\left(\ell^{2 g-2}-1\right)}{\left(\ell^{2}-1\right)(\ell-1)} .
$$

Let $\Theta_{B} \subset J X_{1}$ denote the theta divisor associated to $B$. Then by [9, Lemma 4.3.5], the cardinality $A(\ell)$ of the finite set

$$
\Sigma(\ell):=J X_{1}[\ell] \cap \Theta_{B}
$$

satisfies

$$
A(\ell) \leq \ell^{2 g-2} g(p-1) .
$$

Suppose that there exists an isotropic 2-plane $\Pi \subset \mathbb{F}_{\ell}^{g} \times \mathbb{F}_{\ell}^{g}$ which contains $\leq \ell-2$ points of $\Sigma(\ell)$. Then we can find a pair $(\alpha, \beta)$ satisfying the three properties of the lemma as follows: any nonzero point $x \in \Pi$ determines a line $\left(=\mathbb{F}_{\ell}\right.$-vector space of dimension 1$)$. Since a line contains $\ell-1$ nonzero points, we obtain at most $(\ell-1)(\ell-2)$ nonzero points lying on lines generated by $\Sigma(\ell) \cap \Pi$. Since $(\ell-1)(\ell-2)<\ell^{2}-1$ there exists a nonzero $\alpha$ in the complement of these lines. Now we note that there are $\ell-1$ affine lines parallel 
to the line generated by $\alpha$ and the $\ell$ points on any of these affine lines are of the form $\beta \alpha^{i}$ for $i=0, \ldots, \ell-1$ for some $\beta \in \alpha^{\perp} \backslash\langle\alpha\rangle$. The points $\Sigma(\ell) \cap \Pi$ lie on at most $\ell-2$ such affine lines, hence there exists at least one affine line parallel to $\langle\alpha\rangle$ avoiding $\Sigma(\ell)$. This gives $\beta$.

Finally let us suppose that any isotropic 2 -plane contains $\geq \ell-1$ points of $\Sigma(\ell)$. Then we will arrive at a contradiction as follows: we introduce the set

$$
S=\{(x, \Pi) \mid x \in \Pi \cap \Sigma(\ell) \text { and } \Pi \text { isotropic 2-plane }\} .
$$

with cardinality $|S|$. Then by our assumption we have

$$
|S| \geq(\ell-1) N(\ell)
$$

On the other hand, since any nonzero $x \in \mathbb{F}_{\ell}^{g} \times \mathbb{F}_{\ell}^{g}$ is contained in $\left(\ell^{2 g-2}-1\right) /(\ell-1)$ isotropic 2-planes, we obtain

$$
|S| \leq \frac{\ell^{2 g-2}-1}{\ell-1} A(\ell)
$$

Putting (4.2) and (4.3) together, we obtain

$$
A(\ell) \geq \frac{\ell^{2 g}-1}{\ell+1}
$$

But this contradicts the inequality $A(\ell) \leq \ell^{2 g-2} g(p-1)$ if $\ell \geq g(p-1)+1$.

This completes the proof of Proposition 4.1.

REMARK. - It has been shown [8, Theorem A.6], that $V_{r}$ is dominant for any rank $r$ and any curve $X$, by using a versal deformation of a direct sum a $r$ line bundles.

REMARK. - We note that $V_{r}$ is not separable when $p$ divides the rank $r$ and $X$ is non-ordinary. In that case the Zariski tangent space at a stable bundle $E \in \mathcal{M}_{X_{1}}(r)$ identifies with the quotient $H^{1}\left(X_{1}, \operatorname{End}_{0}(E)\right) /\langle e\rangle$ where $e$ denotes the nonzero extension class of $\operatorname{End}_{0}(E)$ by $\mathcal{O}_{X_{1}}$ given by $\operatorname{End}(E)$. Then the inclusion of homotheties $\mathcal{O}_{X_{1}} \hookrightarrow \operatorname{End}_{0}(E)$ induces an inclusion $H^{1}\left(X_{1}, \mathcal{O}_{X_{1}}\right) \subset$ $H^{1}\left(X_{1}, \operatorname{End}_{0}(E)\right) /\langle e\rangle$ and the restriction of the differential of $V_{r}$ at the point $E$ to $H^{1}\left(X_{1}, \mathcal{O}_{X_{1}}\right)$ coincides with the non-injective Hasse-Witt map.

TOME $135-2007-\mathrm{N}^{\mathrm{O}} 1$ 


\section{Proof of Proposition 1.3}

Since we already know that $\nu(E) \leq(r-1)(2 g-2)$ (see [10,11]) it suffices to show that $\nu(E) \leq(p-1)(2 g-2)$.

We consider the quotient $F^{*} E \rightarrow Q$ with minimal slope, i.e., $\mu(Q)=$ $\mu_{\min }\left(F^{*} E\right)$ and $Q$ semistable. By adjunction we obtain a nonzero morphism $E \rightarrow F_{*}(Q)$, from which we deduce (using Theorem 1.1) that

$$
\mu(E) \leq \mu\left(F_{*} Q\right)=\frac{1}{p}\left(\mu_{\min }\left(F^{*} E\right)+(p-1)(g-1)\right)
$$

hence

$$
\mu\left(F^{*} E\right) \leq \mu_{\min }\left(F^{*} E\right)+(p-1)(g-1) .
$$

Similarly we consider the subbundle $S \hookrightarrow F^{*} E$ with maximal slope, i.e., $\mu(S)=\mu_{\max }\left(F^{*} E\right)$ and $S$ semistable. Taking the dual and proceeding as above, we obtain that

$$
\mu\left(F^{*} E\right) \geq \mu_{\max }\left(F^{*} E\right)-(p-1)(g-1) .
$$

Now we combine both inequalities and we are done.

REMARK. - We note that the inequality of Proposition 1.3 is sharp. The maximum $(p-1)(2 g-2)$ is obtained for the bundles $E=F_{*} E^{\prime}$ (see [3, Theorem 5.3]).

\section{Characterization of direct images}

Consider a line bundle $L$ over $X$. Then the direct image $F_{*} L$ is stable (see [4], Proposition 1.2) and the Harder-Narasimhan filtration of $F^{*} F_{*} L$ is of the form (see [3])

$$
0=V_{0} \subset V_{1} \subset \cdots \subset V_{p-1} \subset V_{p}=F^{*} F_{*} L, \quad \text { with } \quad V_{i} / V_{i-1} \cong L \otimes \omega_{X}^{p-i} .
$$

In particular $\nu\left(F_{*} L\right)=(p-1)(2 g-2)$. In this section we will show a converse statement.

More generally let $E$ be a stable rank-rp vector bundle with $\mu(E)=g-1+\frac{d}{r p}$ for some integer $d$ and satisfying

1) the Harder-Narasimhan filtration of $F^{*} E$ has $\ell$ terms.

2) $\nu(E)=(p-1)(2 g-2)$.

Questions. - Do we have $\ell \leq p$ ? Is $E$ of the form $E=F_{*} G$ for some rank- $r$ vector bundle $G$ ? We will give a positive answer in the case $r=1$ (Proposition 6.1). 
Let us denote the Harder-Narasimhan filtration by

$$
0=V_{0} \subset V_{1} \subset \cdots \subset V_{\ell-1} \subset V_{\ell}=F^{*} E, \quad V_{i} / V_{i-1}=M_{i}
$$

satisfying the inequalities

$$
\mu_{\max }\left(F^{*} E\right)=\mu\left(M_{1}\right)>\mu\left(M_{2}\right)>\cdots>\mu\left(M_{\ell}\right)=\mu_{\min }\left(F^{*} E\right) .
$$

The quotient $F^{*} E \rightarrow M_{\ell}$ gives via adjunction a nonzero map $E \rightarrow F_{*} M_{\ell}$. Since $F_{*} M_{\ell}$ is semistable, we obtain that $\mu(E) \leq \mu\left(F_{*} M_{\ell}\right)$. This implies that $\mu\left(M_{\ell}\right) \geq g-1+d / r$. Similarly taking the dual of the inclusion $M_{1} \subset F^{*} E$ gives a map $F^{*}\left(E^{*}\right) \rightarrow M_{1}^{*}$ and by adjunction $E^{*} \rightarrow F_{*}\left(M_{1}^{*}\right)$. Let us denote

$$
\mu\left(M_{1}^{*}\right)=g-1+\delta,
$$

so that $\mu\left(F_{*}\left(M_{1}^{*}\right)\right)=g-1+\delta / p$. Because of semistability of $F_{*}\left(M_{1}^{*}\right)$, we obtain

$$
-\left(g-1+\frac{d}{r p}\right)=\mu\left(E^{*}\right) \leq \mu\left(F^{*}\left(M_{1}^{*}\right)\right)
$$

hence $\delta \geq-2 p(g-1)-d / r$. This implies $\mu\left(M_{1}\right) \leq(2 p-1)(g-1)+d / r$. Combining this inequality with $\mu\left(M_{\ell}\right) \geq g-1+d / r$ and the assumption $\mu\left(M_{1}\right)-\mu\left(M_{\ell}\right)=(p-1)(2 g-2)$, we obtain that

$$
\mu\left(M_{1}\right)=(2 p-1)(g-1)+\frac{d}{r}, \quad \mu\left(M_{\ell}\right)=g-1+\frac{d}{r} .
$$

Let us denote by $r_{i}$ the rank of the semistable bundle $M_{i}$. We have the equality

$$
\sum_{i=1}^{\ell} r_{i}=r p
$$

Since $E$ is stable and $F_{*}\left(M_{\ell}\right)$ is semistable and since these bundles have the same slope, we deduce that $r_{\ell} \geq r$. Similarly we obtain that $r_{1} \geq r$.

Note that it is enough to show that $r_{\ell}=r$. Since $E$ is stable and $F_{*} M_{\ell}$ semistable and since the two bundles have the same slope and rank, they will be isomorphic.

We introduce for $i=1, \ldots, \ell-1$ the integers

$$
\delta_{i}=\mu\left(M_{i+1}\right)-\mu\left(M_{i}\right)+2(g-1)=\mu\left(M_{i+1} \otimes \omega\right)-\mu\left(M_{i}\right) .
$$

Then we have the equality

$$
\sum_{i=1}^{\ell-1} \delta_{i}=\mu\left(M_{\ell}\right)-\mu\left(M_{1}\right)+2(\ell-1)(g-1)=2(\ell-p)(g-1) .
$$

We note that if $\delta_{i}<0$, then $\operatorname{Hom}\left(M_{i}, M_{i+1} \otimes \omega\right)=0$. 
Proposition 6.1. - Let $E$ be stable rank-p vector bundle with

$$
\mu(E)=g-1+d / p \quad \text { and } \quad \nu(E)=(p-1)(2 g-2) .
$$

Then $E=F_{*} L$ for some line bundle $L$ of degree $g-1+d$.

Proof. - Let us first show that $\ell=p$. We suppose that $\ell<p$. Then

$$
\sum_{i=1}^{\ell-1} \delta_{i}=2(\ell-p)(g-1)<0
$$

so that there exists a $k \leq \ell-1$ such that $\delta_{k}<0$. We may choose $k$ minimal, i.e., $\delta_{i} \geq 0$ for $i<k$. Then we have

$$
\mu\left(M_{k}\right)>\mu\left(M_{i}\right)+2(g-1) \text { for } i>k .
$$

We recall that $\mu\left(M_{i}\right) \leq \mu\left(M_{k+1}\right)$ for $i>k$. The Harder-Narasimhan filtration of $V_{k}$ is given by the first $k$ terms of the Harder-Narasimhan filtration of $F^{*} E$. Hence $\mu_{\min }\left(V_{k}\right)=\mu\left(M_{k}\right)$.

Consider now the canonical connection $\nabla$ on $F^{*} E$ and its first fundamental form

$$
\phi_{k}: V_{k} \longleftrightarrow F^{*} E \stackrel{\nabla}{\longrightarrow} F^{*} E \otimes \omega_{X} \longrightarrow\left(F^{*} E / V_{k}\right) \otimes \omega_{X} .
$$

Since $\mu_{\min }\left(V_{k}\right)>\mu\left(M_{i} \otimes \omega\right)$ for $i>k$ we obtain $\phi_{k}=0$. Hence $\nabla$ preserves $V_{k}$ and since $\nabla$ has zero $p$-curvature, there exists a subbundle $E_{k} \subset E$ such that $F^{*} E_{k}=V_{k}$.

We now evaluate $\mu\left(E_{k}\right)$. By assumption $\delta_{i} \geq 0$ for $i<k$. Hence

$$
\mu\left(M_{i}\right) \geq \mu\left(M_{1}\right)-2(i-1)(g-1) \quad \text { for } i \leq k,
$$

which implies that

$$
\operatorname{deg}\left(V_{k}\right)=\sum_{i=1}^{k} r_{i} \mu\left(M_{i}\right) \geq \operatorname{rk}\left(V_{k}\right) \mu\left(M_{1}\right)-2(g-1) \sum_{i=1}^{k} r_{i}(i-1) .
$$

Hence we obtain

$$
p \mu\left(E_{k}\right)=\mu\left(V_{k}\right) \geq \mu\left(M_{1}\right)-2(g-1) C,
$$

where $C$ denotes the fraction $\left(\sum_{i=1}^{k} r_{i}(i-1)\right) / \mathrm{rk}\left(V_{k}\right)$. We will prove in a moment that $C \leq \frac{1}{2}(p-1)$, so that we obtain by substitution

$$
p \mu\left(E_{k}\right) \geq(2 p-1)(g-1)+d-(g-1)(p-1)=p(g-1)+d=p \mu(E),
$$

contradicting stability of $E$. Now let us show that $C \leq \frac{1}{2}(p-1)$ or equivalently

$$
\sum_{i=1}^{k} i r_{i} \leq \frac{1}{2}(p+1) \sum_{i=1}^{k} r_{i}
$$


But that is obvious if $k \leq \frac{1}{2}(p-1)$. Now if $k>\frac{1}{2}(p-1)$ we note that passing from $E$ to $E^{*}$ reverses the order of the $\delta_{i}$ 's, so that the index $k^{*}$ for $E^{*}$ satisfies $k^{*} \leq \frac{1}{2}(p-1)$. This proves that $\ell=p$.

Because of (6.1) we obtain $r_{i}=1$ for all $i$ and therefore $E=F_{*} M_{p}$.

\section{Stability of $F_{*} E$ ?}

Is stability also preserved by $F_{*}$ ?

We show the following result in that direction.

Proposition 7.1. - Let $E$ be a stable vector bundle over $X$. Then $F_{*} E$ is simple.

Proof. - Using relative duality $\left(F_{*} E\right)^{*} \cong F_{*}\left(E^{*} \otimes \omega_{X}^{1-p}\right)$ we obtain

$$
H^{0}\left(X_{1}, \operatorname{End}\left(F_{*} E\right)\right)=H^{0}\left(X, F^{*} F_{*} E \otimes E^{*} \otimes \omega_{X}^{1-p}\right) .
$$

Moreover the Harder-Narasimhan filtration of $F^{*} F_{*} E$ is of the form (see [3])

$$
0=V_{0} \subset V_{1} \subset \cdots \subset V_{p-1} \subset V_{p}=F^{*} F_{*} E, \quad \text { with } \quad V_{i} / V_{i-1} \cong E \otimes \omega_{X}^{p-i} .
$$

We deduce that

$$
H^{0}\left(X, F^{*} F_{*} E \otimes E^{*} \otimes \omega_{X}^{1-p}\right)=H^{0}\left(X, V_{1} \otimes E^{*} \otimes \omega_{X}^{1-p}\right)=H^{0}(X, \operatorname{End}(E)),
$$

and we are done.

\section{BIBLIOGRAPHY}

[1] A. Beauville - "On the stability of the direct image of a generic vector bundle", preprint available at http://math.unice.fr/〜beauvill/pubs/ imdir.pdf.

[2] G. Faltings - "Projective connections and G-bundles", J. Algebraic Geom. 2 (1993), p. 507-568.

[3] K. Joshi, S. Ramanan, E. Z. Xia \& J. K. YU - "On vector bundles destabilized by Frobenius pull-back", Compos. Math. 142 (2006), p. 616630.

[4] H. Lange \& C. Pauly - "On Frobenius-destabilized rank-2 vector bundles over curves", Comm. Math. Helvetici 83 (2008), p. 179-209.

[5] Y. Laszlo \& C. PAuly - "The Frobenius map, rank 2 vector bundles and Kummer's quartic surface in characteristic 2 and 3", Adv. Math. 185 (2004), p. 246-269. 
[6] J. Le Potier - "Module des fibrés semi-stables et fonctions thêta", in Moduli of vector bundles (Sanda 1994, Kyoto 1994), Lect. Notes Pure Appl. Math., vol. 179, Dekker, New York, 1996, p. 83-101.

[7] V. B. Mehta \& S. Subramanian - "Nef line bundles which are not ample", Math. Z. 219 (1995), p. 235-244.

[8] B. Osserman - "The generalized Verschiebung map for curves of genus 2", Math. Ann. 336 (2006), p. 963-986.

[9] M. Raynaud - "Sections des fibrés vectoriels sur une courbe", Bull. Soc. Math. France 110 (1982), p. 103-125.

[10] N. I. ShEPHERD-BARRON - "Semistability and reduction $\bmod p$ ", Topology 37 (1998), p. 659-664.

[11] X. Sun - "Remarks on semistability of $G$-bundles in positive characteristic", Compos. Math. 119 (1999), p. 41-52. 